\title{
Air polishing and CHX effects in addition to subgingival ultrasonic instrumentation on clinical periodontal parameters: A randomised clinical trial
}

\author{
Ayse Caygur
}

Department of Periodontolgy, Faculty of Dentistry, Near East University, Nicosia, Cyprus.

Corresponding author: Ayse Caygur (E mail Address: ayse.caygur@gmail.com )

(Submitted: 04 September 2020 - Revised version received: 23 September 2020 - Accepted: 11 - October 2020 - Published online: 26 December 2020)

\begin{abstract}
Objectives: This study was performed to investigate the efficacy of using glycine powder air-polishing (GPAP) or antiseptics (chlorhexidine, (HX) adjunctively to scaling and root planing (SRP) in the treatment of periodontitis.

Methods: In this trial, 90 patients (between 28 and 80 years old) who had at least three teeth with 3- to 7-mm periodontal pockets were included from the Department of Periodontology. The patients were divided into three groups randomly. In the control group, ultrasonic instrumentation was performed with distilled water and hand instrumentation. In the CHX group, ultrasonic instrumentation was performed with CHX and hand instrumentation. In the GPAP group, in addition to SRP with ultrasonic performed with distilled water and hand instrumentation. GPAP was performed for 10 s per periodontal pocket using a Perio-Flow device. All treatments were performed in one session.

Resuls: The scores of plaque index (PI), gingival index (GI), probing depth (PD), bleeding on probing (BOP), and clinical attachement level (CAL) were decreased in all groups after 1 month, and the results were statistically significant in intergoup comparisions. PI and CAL scores were statistically significant in intragroup comparisions in GPAP group. Altough GI, PD, and BOP scores were lower in the GPAP group than $\mathrm{CHX}$ and control group, the differences after 1 month were not statistically significant.

Conclusion: Within the limits of this study, it was seen that SRP was effective alone in the treatment of periodontitis. However, using a CHX with ultrasonic devices has little beneficial effects on periodontal parameters when compared with control and GPAP groups. GPAP groups was found more effectively in PI and CAL scores.

Keyword: Dental plaque, root planning, chlorhexidine, glycine, periodontal index
\end{abstract}

\section{Introduction}

Periodontitis affects the soft and mineralized tissues surrounding the tooth. It is a disease characterized by a decrease in the level of alveolar bone and attachment caused by subgingival biofilm that contains periodontal pathogenic microorganisms. ${ }^{1}$ The most important part of the treatment of periodontitis is scaling and root planing (SRP). SRP generally aims to eliminate subgingival microorganisms and remove bacterial endotoxins which penetrated into the cementum surface, thereby controlling the progression of periodontal destruction creating a healthy subgingival enviroment. ${ }^{2}$

The function of SRP is to reduce the bacterial population in the subgingival flora, inflammation, pocket depth, and clinical attachment level. ${ }^{3}$ However, as the depth of the pocket increases, the effectiveness of these methods may decrease. ${ }^{4}$ Therefore, there is a need for methods to be applied in addition to the SRP process performed with ultrasonic and hand tools. ${ }^{5}$

Antimicrobial agents have been developed for use with SRP to delay recurrence of colonization with existing pathogens, and improved clinical results have been observed in the evaluations made in the treated area. ${ }^{6}$

One of these antimicrobial agent is chlorhexidine belonging to the biguanide family, and are widely used in dental and medical treatments as an antiseptic agent. It is a broad-spectrum antiseptic effective on a variety of Gram positive and Gram negative bacteria as well as yeasts and viruses. ${ }^{7}$ The ability of chlorhexidine (CHX) to adhere to the dental pellicle and oral mucosa extends its anti-plaque effect. ${ }^{8}$
CHX mouthwash (0.2\%) are widely used before surgical applications, but can also be used as an irrigant in addition to mechanical instrumentation at varying concentrations. Besides these effects of CHX, there are many side effects. One of the common side effects of CHX is brown discoloration of teeth, some restorative materials, and dorsum of the tongue. ${ }^{9}$ Histological studies have shown that CHX may lead to delay in tissue healing processes by causing inhibition of gingival fibroblast proliferation depending on the in-vitro dose, and other studies with fibroblast cultures have explained that it can be severely cytotoxic at concentrations between 17 and 100 $\mathrm{mM}{ }^{10,11}$ Ribeiro et al, in their study on rats, showed that the use of chlorhexidine digluconate caused primary DNA damage in leukocytes and oral mucosal cells. ${ }^{12}$

Air-polishing devices (APDs) are highly effective in removing plaque and extrinsic staining by generating a slurry of pressurized air, water, and abrasive powder. For this purpose, fine-grained (DV90: $63 \mathrm{~mm}$ ) glycine powder applied directly to the periodontal pocket has been shown to be effective and safe in removing subgingival biofilm from periodontal pockets. ${ }^{13}$ Glycine means sweet in ancient Greek. Non-essential amino acid and polypeptide are important components of glycine. It is odorless, colorless, non-allergenic, and dissolves quickly in water. Also, it has anti-inflammatory, immunomodulatory, and cytoprotective (cell protective) effects. ${ }^{14}$ In one study, glycine powder air-polishing (GPAP) reduced the total viable bacterial counts in periodontal pockets with probing depth (PDs) ranging from 3 to $5 \mathrm{~mm}$ and to a significantly greater extent than SRP using curettes. ${ }^{15}$ However, no studies 
have investigated the efficacy of using GPAP adjunctively or antiseptics (CHX) with SRP in the treatment of periodontal parameters. Therefore, the aim of this clinical study was to evaluate the effect of using GPAP or CHX adjunctively with SRP on periodontal parameters.

\section{Materials and Methods}

Ninety (90) patients (28-80 years of age) who applied to Periodontology Department and had periodontal pockets between 3 and $7 \mathrm{~mm}$ in at least three teeth were included in this study.

Patients with known pregnancy, lactation, and systemic disease (diabetes), those who have taken antibiotics in the last 4 months or have used other drugs permanently, and individuals who have undergone any periodontal treatment within 6 months and those with furcha defect were not included.

\section{Periodontal Examination}

The plaque index (PI), ${ }^{16}$ gingival index (GI), ${ }^{17}$ clinical attachment level (CAL), PD and bleeding on probing (BOP) were measured at baseline and 1 month after treatment by a single calibrated examiner who was not aware of the type of treatment applied. The PI, GI, PD, position of the gingival margin, and BOP were evaluated with a periodontal probe at six sites on all teeth. BOP was assessed by the percentage of sites that bled after probing.

\section{Study Design}

This was a single-blinded, computer-randomized, controlled clinical study. Written informed consent was obtained from each study participant after all procedures had been fully explained. This study was approved by the Ethics Committee (2015/33-236). All patients had previously undergone and completed initial periodontal therapy. The patients were divided into three groups. In the control group, ultrasonic instrumentation (Piezonmaster 700; Electro Medical Systems, Nyon, Switzerland) was performed with distilled water and hand instrumentation. In the first group, ultrasonic instrumentation was performed with CHX (Drogsan, Istanbul, Turkey, $0.2 \%$ ) and hand instrumentation. In the second group, in addition to SRP with ultrasonic performed with distilled water and hand instrumentation, GPAP (Air-Flow Perio Powder; Electro Medical Systems) was performed for $10 \mathrm{~s}$ per periodontal pocket using a Perio-Flow device (Air-Flow Master; Electro Medical Systems). All treatments were performed in one session.

\section{Statistical Analysis}

For all three groups, the mean values of the clinical parameters were calculated. To evaluate the changes over time within each group, one-way repeated ANOVA was used. Levene Test was used to evaluate the homogeneity of variances of the values of $P>0.05$ ANOVA test, if the values of $P \leq 0.05$ Kruskal-Wallis Variance Analysis was used. A $t$-test was used for comparison among groups at each time.

\section{Results}

All 90 patients were involved in a 1-month study period. The standard deviations and mean of the PI, GI, PD, BOP, GR, and CAL in the first, second, and control groups are presented in Table 1. The scores of PI, GI, PD, BOP, and CAL were

\begin{tabular}{|c|c|c|}
\hline & Baseline & 1 month \\
\hline \multicolumn{3}{|l|}{$\mathrm{PI}$} \\
\hline CHX group & $0,920,73$ & $0,280,40^{*}$ \\
\hline GPAP group & $1,610,82$ & $0,410,52^{*}$ \\
\hline Control group & $0.63 \pm 0.74$ & $0.39 \pm 0.58^{*}$ \\
\hline \multicolumn{3}{|l|}{$\mathrm{Gl}$} \\
\hline CHX group & $1.42 \pm 0.95$ & $0.81 \pm 0.40^{*}$ \\
\hline GPAP group & $1,56 \pm 0,70$ & $0.40 \pm 0.51^{*}$ \\
\hline Control group & $1.80 \pm 0.78$ & $0.76 \pm 0.71^{*}$ \\
\hline \multicolumn{3}{|l|}{ PD } \\
\hline CHX group & $4.78 \pm 0.57$ & $3.78 \pm 0.61^{*}$ \\
\hline GPAP group & $4.72 \pm 0.49$ & $3.50 \pm 0.34^{*}$ \\
\hline Control group & $4.90 \pm 0.68$ & $3.94 \pm 0.93^{*}$ \\
\hline \multicolumn{3}{|l|}{ BOP } \\
\hline $\mathrm{CHX}$ group & $0.57 \pm 0.25$ & $0.13 \pm 0.22^{*}$ \\
\hline GPAP group & $0.44 \pm 0.11$ & $0.04 \pm 0.08^{*}$ \\
\hline Control group & $0.44 \pm 0.21$ & $0.08 \pm 0.19^{*}$ \\
\hline \multicolumn{3}{|l|}{ CAL } \\
\hline $\mathrm{CHX}$ group & $0.98 \pm 0.86$ & $0.64 \pm 0,80^{*}$ \\
\hline GPAP group & $1.62 \pm 1.43$ & $0,89 \pm 1,12^{*}$ \\
\hline Control group & $0.93 \pm 0.71$ & $0.11 \pm 0.23^{*}$ \\
\hline
\end{tabular}

Data are presented as mean \pm standard deviation.

*Differences between T0 and T4 were statistically significant in the intergroup comparisons for both groups; $\mathrm{P}<0.05$.

decreased in all groups on 1 month and was statistically significant in intergoup comparisions. PI and CAL scores were statistically significant in intragroup comparisions in second group. Altough GI, PD, and BOP scores were lower in the second group than first and control group, the differences on 1 month were not statistically significant.

\section{Discussion}

Dental plaque in the periodontal pocket and on the root surface was recently shown that cause changes in biological structure. Bacterial exotoxins that penetrate the root surface cause changes of antibody complexes, and microbial metabolism. ${ }^{18}$ The efficacy of periodontal therapy is directly related to the percentage of bacteria in the pocket. ${ }^{19}$ Mechanical debridement is arguably the most important part of treating periodontal disease. Studies have shown that the effectiveness of mechanical debridement is superior to measurable indexes. These end points include CAL, PD, BOP, and alterations in the subgingival microflora. ${ }^{4}$ Due to anatomical limitations, it is not always possible to reach the root surface and perform effective debridement. ${ }^{20}$ For this reason, new searches have been started.

Petersilka et al, ${ }^{21}$ demostrated that, up to $30 \%$ of the total surface area of treated roots can be reinfected with pathogenic bacteria, therfore influencing the reoccurence of periodontal disease. Yilmaz and Bayindir, ${ }^{22}$ used essential oils and CHX as a cooling agent and found that there were significant reduction in BOP, GI, PD, and PI in all groups on 1 month. The difference among the groups were not significant at any point except in BOP scores. On the other hand, a similar study by Tumer et $\mathrm{al},{ }^{8}$ there was a significant decrease in all parameters but there were not statistically significant difference between the groups. 
Guarnelliet al., ${ }^{23}$ demonstrated that the use of CHX adjunctive to UMI in patients with aggressive periodontitis, indicated no additional efficacy over UMI alone. Similar with this report, in our study, the reduction of the clinical parameters in the CHX group was not statistically significant when compared with the other groups. Also, the effectiveness of the antimicrobial agents may be affected due to the contamination of antimicrobial agents with blood and gingival crevicular fluid contents. ${ }^{24}$

The present study revealed that mechanical instrumentation and GPAP had the same effect on PI scores when used in periodontal pockets with moderate PD. Similarly, Flemmig et al. ${ }^{25}$ showed that hand instrumentation and GPAP had the same effect on PI when used in periodontal pockets with PDs of up to approximately $3-5 \mathrm{~mm}$. Because the use of low-abrasive powder led to a significantly higher reduction in subgingival bacteria than hand instrumentation, it may be speculated that the clinical outcomes of periodontal maintenance therapy using subgingival air polishing may be equivalent to or even better than the clinical outcomes of conventional modes of debridement. ${ }^{26}$ In our study, all scores decreased in all groups, but the difference between the groups was not statistically significant exept in PI and CAL. PI and CAL scores were statistically significant in intragroup comparisions in GPAP group. In some studies, it has been shown that it fails to remove subgingival calculus and is effective in bacterial elimination due to its low abrasiveness. Although bleeding decreased after both treatments, better results were obtained with glycine. ${ }^{27}$ On the other hand, it was determined that it caused a decrease in both the removal of the biofilm and the recolonization of bacteria (P. gingivalis) in the applied area. On the contrary, Muller et al. ${ }^{28}$ also found a significant decrease in the PD and BOP in their ultrasonic and Perio-Flow groups when used with SRP at 3-month intervals, but no significant difference was found between the two groups. GPAP has been shown to be more efficient in debriding deep periodontal sites than SRP using curettes. Similar to this study, Caygur et al..$^{20}$ found no statistically significant difference between the control group and the GPAPl group, although there was a significant decrease in periodontal parameters in the GPAP group in their study.

\section{Conclusion}

Within the limits of this study, it was seen that SRP was effective alone in the treatment of periodontitis. However, using a CHX with ultrasonic devices has little beneficial effects on periodontal parameters when compared with control and GPAP groups. GPAP groups was found more effectively in PI and CAL scores.

\section{Acknowledgments}

None.

\section{References}

1. American Academy of Periodontal. Glossary of Periodontal Terms,4th Ed Chicago: American Academy of Periodontology. 2001; 40

2. Nagarakantı S, Gunupatı S, Chava VK, Reddy BVR. Effectivess of subgingival irrigation as an adjunt to scaling and root planing in the treatment of chronic periodontitis: A systematic review. JCDR. 2015;9(7):6-8.
3. Ioannou I, Dimitriadis N, Papadimitrious K, et al. Hand instrumentation versus ultrasonic debridement in the treatment of chronic periodontitis: A randomized clinical and microbiological trial. J Clin Periodontol. 2009;36:132-141.

4. Cobb CM. Clinical significance of non-surgical periodontal therapy: An evidence-based perspective of scaling and root planing. J Clin Periodontol. 2002;2: 6-16

5. Quirynen M, Teughels W, De Soete M, et al. Topical antiseptics aspects. Periodontol 2000. 2002:28:72-92

6. Cosyn, J, Sabzevar MM. A systematic review on the effects of subgingival chlorhexidine gel administration in the treatment of chronic periodontitis. J Periodontol. 2005;76:1805-13.

7. Gunsolley JC. Clinical efficacy of antimicrobial mouthrinses. J Dent. 2010;38:6-10.

8. Tumer $\mathrm{H}$, Berberoglu A, Caygur A, et al. Clinican evaluation of chlorhexidine and essential oils adjunctive effects in subgingival ultrasonıc Instrumentation on periodontal parameters and halitosis. J Essent Oils Bearing Plants. 2019:22(1):169-175.

9. Walker CB, Karpani K, Baehn, P. Chemotherapeutics: Antibiotics and other antimicrobials. Periodontol 2000. 2004;36:146-165.

10. Dogan S, Gunay H, Leyhausen G, et al. Effect of low-concentrated chlorhexidine on growth of Streptococcus sobrinus and primary human gingival fibroblasts. Clin Oral Investig. 2003;7(4):212-216.

11. Boisnic S, Ben Slama L, Branchet-Gumila MC, et al Wound healing effect of Eludril in a model of human gingival mucosa(Serbian). Rev Stomatol Chir Maxillofac. 2006;107:431-35.

12. Ribeiro DA, Bazo AP, da Silva Franchis CA, et al. Chlorhexidine induces DNA damage in rat peripheral leukocytes and oral mucosel cells. J Periodontal Res. 2004:39(5):358-361.

13. Petersilka G, Faggon CM, Stratmann U, et al. Effect of glycine powder airpolishing on the gingiva. J Clin Periodontol. 2008; 35:324-332.

14. Zhong Z, Wheeler MD, Li X, et al L-Glycine: A novel antiinflammatory, immunomodulatory, and cytoprotective agent. Curr Opin Clin Nutr Metab Care. 2003; 6:229-240

15. Petersılka GJ. Subgingival air-polishing in the treatment of periodonta biofilm infections. Periodontol 2000. 2011:55:124-142.

16. Silness J, Loe H. Periodontal disease in pregnancy II. Correlation between oral hygiene and periodontal condition. Acta Odontol Scand. 1964;22:121-135.

17. Loe H, Silness J. Periodontal disease in pregnancy I. Prevalance and severity. Acta Odontol Scand. 1963:21:533-551.

18. Khosravi M, Baharami ZS, Atabaki MSJ. et al. Comparative effectiveness of hand and ultrasonic instrumentations in root surface planing in vitro. J Clin Periodontol. 2004;31:160-165.

19. Chaves ES, Jeffcoat MK, Ryerson CC. et al. Persistent bacterial colonization of Porphymonas gingivalis, Prevotella intermedia, and Actinobacillus actinomycetemcomitans in periodontitis and its association with alveolar bone loss after 6 months of therapy. J Clin Periodontol. 2000;27:897-903.

20. Caygur A, Albaba MR, Berberoglu A, et al. Efficacy of glycine powder air-polishing combined with scaling and root planing in the treatment of periodontitis and halitosis: A randomised clinical study. 2017:45(3):1168-1174.

21. Petersilka GJ, Ehmke B, Flemming TF. Antimicrobial effects of mechanical debriment. Periodontol 200. 2002;28: 56-71.

22. Yilmaz HG, Bayındır H. Clinical evaluation of chlorhexidine and essential oils for adjuctive effects in ultrasonic instrumentation of furcation involvements: A randomized control trial. J Clin Periodontol. 2011;38:637-643

23. Guarnelli ME, Franceschetti G, Manfrini R. et al. Adjuctive effect of chlorhexidine in ultrasonic instrumentation of aggresive periodontitis patients: a pilot study. J Clin Periodontol. 2008:35:333-341.

24. Preus HR, Koldsland OC, Aass AN, et al. The plaque-and-gingivitis-inhibiting capacity of a commercially available essential oil product. A parallel, splitmouth, single blind,randomized, placebo-controlled clinical study. Acta Odontol Scand. 2013;71:1613-19.

25. Flemming TF, Hetzel $M$, Topoll $H$, et al. Subgingival debridement efficacy of glycine powder air polishing. J Periodontol. 2007;78:1002-1010

26. Petersilka GJ, Tunkel J, Barakos K, et al. Subgingival plaque removal at interdental sites using a low abrasive air polishing. J Periodontol. 2003;74:307-311.

27. Moene R, Decaillet F, Andresan E, et al. Subgingival plaque removal using new air-polishing device. J Periodontol. 2010; 81:79-88.

28. Müller N, Moene R, Cancela JA, et al. Subgingival air polishing with eryhriol during periodontal maitenanace. J Clin Periodontol. 2014;41:883-889.

This work is licensed under a Creative Commons Attribution-NonCommercial 3.0 Unported License which allows users to read, copy, distribute and make derivative works for non-commercial purposes from the material, as long as the author of the original work is cited properly. 